\title{
Tomography of Accretion Flows in Binary Stars and Active Galactic Nuclei
}

\author{
Keith Horne \\ Physics $6 \sigma^{\circ}$ Astronomy, North Haugh, St. Andrews KY16 9SS, Scotland, \\ UK, Email: kdh1@st-and.ac.uk
}

\begin{abstract}
.
Astrotomography resolves accretion flows with micro-arcsecond angular resolution. Eclipses by a binary companion star slice up a disk surface, giving monochromatic maps of the disk, or spectra from any region of its surface. Doppler tomography maps emission-line regions from the changing velocity profile as the binary rotates, revealing radial and azimuthal structure, gas streams, irradiated companion stars, magnetic flows, and slingshot prominences. Echo mapping exploits time delays between the hard radiation from near the compact object at the focus of the flow, and softer emission generated by irradiation of regions farther out. The maximum entropy techniques for fitting intensity maps to data are currently being extended by incorporating local physics and mapping physical parameters such as temperature, density, surface density, and velocity dispersion.
\end{abstract}

\section{Astrotomography}

Astrotomography refers to a suite of indirect imaging techniques that are being used to make pictures of astronomical objects that are too small and too far away to be resolved by direct imaging. The techniques can be used for a limited but interesting range of astronomical objects, including rotating stars and planets, binary star systems, particularly those with with accretion flows, and the gas flows around the nuclei of active galaxies. The resolution achieved in current astrotomography experiments is typically $\sim 10^{-6}$ arcseconds.

Tomography refers to reconstructing an image from measurements of projections, or "slices" of that image. Tomography is well established and very important in medical imaging, where rotating scanners pass X-rays or ultra-sonic waves through a patient's body to obtain the projections needed to reconstruct images inside the body, enabling medical diagnosis without surgery.

Unlike medicine, Astronomy is a "hands off" science. We cannot place our objects into a scanner to obtain the required projections. For Astrotomography, we resort to various "tricks" to obtain the projections. Assisted by rotation of star, planet, or binary star system, we use occultations of one part of the system by another, doppler shifts arising from the rotation and from the gas flows within the system, and finally time delays arising when emission from one part of the system stimulates emission in another. 
Astrotomography problems are more difficult than medical tomography because the observable projections are weighted line integrals following curved paths across the image of interest, and in some cases the data provide only 1 or 2 projections to constrain the image, rather than a complete set. The resulting ambiguity can fortunately be treated by maximum entropy fitting techniques, which locate the "simplest" image that fits the data, steering the solution toward a smooth image, or some preferred symmetry.

This paper surveys the main techniques and presents illustrative results from Astrotomography experiments involving accretion flows in binary stars and active galactic nuclei. The selection is biased toward work in which the author has played a direct role, and should not be mistaken for a complete survey of the field. For accretion flows in binary systems, the main techniques are eclipse mapping (Horne 1985, 1993) for continuum radiation (Section 2), and Doppler tomography (Marsh \& Horne 1988) for emission lines (Section 3). For active galaxies, Echo mapping (Blandford \& Mc Kee 1982, Horne 1994) resolves emission-line regions using time delays and Doppler shifts (Section 4). New developments incorporate local physics to map physical parameters rather than intensities (Section 5).

\section{Eclipse Mapping}

Eclipse mapping (Horne 1985, 1993) interprets the eclipse lightcurves of a binary system in which an accretion disk flow is being occulted by the binary companion star. In bright states the accretion disk can dominate the optical and ultraviolet light of the system, producing a broad roughly gaussian eclipse in the lightcurve. From the observed shapes of these eclipses, plus a handful of parameters specifying the eclipse geometry, we recover surface brightness maps of the accretion disk.

A computer model of the binary is used to compute synthetic eclipse lightcurves. The companion star is assumed to fill its Roche lobe. Material flows through the nozzle in the Roche potential at the inner Lagrangian point (L1) into a ballistic gas stream that feeds into the accretion disk (Lubow \& Shu 1975). This supersonic flow should remain close to the orbital plane $\left(Z / R \sim C_{s} / V_{K e p}<<1\right)$, so we model it with a 2-dimensional flat or concave surface in or just above the orbital plane, sometimes including an outward-facing rim.

Dividing the disk surface into discrete elements, each with a position $\vec{X}$, intensity $I_{\nu}(\vec{X})$, and solid angle $d \Omega(\vec{X})$, the synthetic lightcurve is obtained by summing up contributions from all visible surface elements

$$
f_{\nu}(\phi)=\int I_{\nu}(\vec{X}) O(\vec{X}, \phi) d \Omega(\vec{X})+f_{0} .
$$

Here $O(\vec{X}, \phi)$ is the fraction of surface element $\vec{X}$ visible to the observer at phase $\phi$, and $f_{o}$ is a constant background flux arising from the companion star and any other sources of light that are not eclipsed.

Parameters defining the eclipse geometry are best established by observing eclipses during a quiescent state, when the accretion flow itself is relatively faint and the main light sources are the compact object at the center of the disk, and 
the "hotspot" where the gas stream from the companion star collides with the outer rim of the disk (Wood et al. 1986, 1989). By timing the phases of ingress and egress of the central object and hotspot (4 constraints), we determine 4 parameters, the mass ratio $q$, inclination $i$, phase of conjunction $\phi_{0}$, and disk radius $R_{D} / a$. With the eclipse geometry established, we can compute ingress and egress phases for any point on the disk surface and hence evaluate $O(\vec{X}, \phi)$.

Eclipse mapping must cope with far less complete information than in medical tomography. Eclipses offer only 2 projections, ingress and egress. Moreover, the derivative of the lightcurve is a weighted line integral along the curved edge of the occulted region, with negative weights on the ingress branch and positive weights on the egress branch. A maximum entropy fitting technique is therefore required to invert the eclipse lightcurve and recover a map of the disk surface (Horne 1985, Baptista \& Steiner 1993). In fitting a two-dimensional map to a one-dimensional lightcurve, there are an infinite number of solutions. This ambiguity is resolved by using the entropy to "steer" the solution toward an axi-symmetric map, yielding the most nearly axi-symmetric map that fits the data. The radial structure is thereby determined by the eclipse data, but any azimuthal structure, such as the hotspot, becomes smeared in azimuth by the entropy.

Observing eclipses and constructing disk maps at several wavelengths opens an approach to the observational study of accretion disk atmospheres, which differ from stellar atmospheres in several important respects (large velocity gradients, gravity increasing with height, finite optical depth, energy generation within the atmosphere). From broad-band eclipse mapping, color-color and color-magnitude diagrams show that disk surfaces in the high state are opaque thermal radiators with vertical temperature gradients steeper than blackbodies but less steep than model atmospheres (Horne \& Cook 1985, Horne \& Stiening 1985, Rutten et al. 1992, Bruch et al. 1996)

Distances are found by applying a vertical shift in the color-magnitude diagrams, a method analogous to main-sequence fitting of star clusters. Actually, the vertical shift determines the angular scale parameter $\theta=R_{L 1} \sqrt{\cos i} / D$, and the distance $D$ follows by combinating this with a linear scale from the binary period and an observed velocity. The surface brightness maps are then expressed as brightness temperature maps to test the theoretical temperature-radius profile for steady-state disks

$$
T_{e f f}^{4} \propto \frac{M \dot{M}}{R^{3}} .
$$

This relationship holds up well for the disks in short-period dwarf novae in outburst and for longer-period nova-like variables, giving mass accretion rate estimates good to factors of $\sim 3$ (Horne \& Cook 1985, Horne \& Stiening 1985, Rutten et al. 1992).

In quiescent dwarf novae, however, optical brightness temperatures are to first order independent of radius (Wood et al. 1986, 1989). This is qualitatively consistent with disk instability theories, in which the disk structure is frozen by low viscosity in quiescence, and represents a fossil remnant of conditions prevailing at the end of the previous outburst. Current work aims to map the physical structure of quiescent disks, the temperature, surface density, and 
turbulence as functions of radius, by fitting to eclipses of the optically-thin emission (Vrielmann, these proceedings).

In many nova-like variables, particularly those with orbital periods of 3 to 4 hours, the eclipse maps reveal temperature-radius profiles flatter than expected (Rutten et al. 1992). The flat profiles arise from "V-shaped" eclipse lightcurves. This "eclipse shape anomaly" is a major problem in the field, and may signal some mixture of important physical processes not included in the simplest disk theories ( e.g. non-radiative cooling via global magnetic field and/or wind extraction of energy from the inner disk ) or break-down of the disk mapping assumptions ( high disk rim blocking inner disk, inner-disk being burried behind opaque wind photosphere).

Multi-wavelength eclipse mapping has been pressed to its natural limit by observing spectra as functions of time thru eclipses. The resulting stack of monochromatic eclipse maps yields the spectrum from any desired region of the disk surface, revealing absorption and emission line fluxes as well as continuum slopes and intensities as functions of radius. In the UX UMa disk, Balmer absorption lines in the inner disk turn over into emission in the outer disk (Rutten et al. 1994). Eclipse mapping with HST extends these results into the ultraviolet (Baptista et al. 1995). This method will bring tight constraints to bear in tests of disk model atmospheres.

\section{Doppler Tomography}

Emission lines with double-peaked velocity profiles are seen in the spectra of quiescent accretion disks. These arise from the Keplerian orbital motion of gas circulating in the disk. Because the flow is supersonic, each velocity bin in the line profile arises from a distinct region of the disk surface. Lines of constant Doppler shift form a dipole pattern on the face of the accretion disk (Horne \& Marsh 1986). The splitting of the two peaks reflects the projected Kepler velocity at the outer rim of the disk. The observed shape of the emission-line wings constrains the radial structure of the disk's emission-line region.

Doppler tomography (Marsh \& Horne 1988) maps emission-line regions by regarding the Doppler profile as a projection of the velocity field along the line of sight. It relies on rotation of the binary to deliver a set of projections in different directions as the line of sight rotates with respect to the flow. Given time-resolved spectra $f(v, \phi)$, with suitable resolution in radial velocity $v$ and binary phase $\phi$, the 2-dimensional constraints from half a binary orbit suffice to construct a 2-dimensional map of the system in Doppler coordinates $\left(v_{x}, v_{y}\right)$.

The Doppler coordinates simply label each part of the flow with a velocity vector $\left(v_{x}, v_{y}\right)$ rather than a position vector $(x, y)$. They are convenient because the contours of constant Doppler shift are straight lines across the Doppler map, thus projections in different directions yield the velocity profiles at different phases. The Doppler map is therefore closely related to the data, and in fact we can use the same algorithm employed in medical imaging to calculate Doppler maps from trailed-spectrogram data.

Doppler maps give distorted but recognizable pictures of the accretion flow (Fig. 1). The uniformly-rotating companion star retains the "teardrop" shape of the Roche lobe, but rotated by $90^{\circ}$ so that its center is at $\left(0,+K_{2}\right)$. Each 

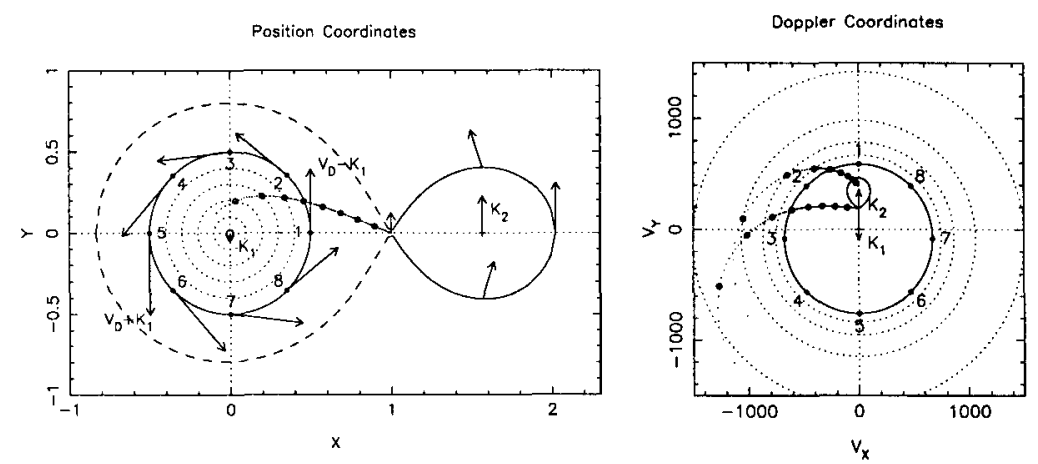

Figure 1. Comparison of position and Doppler coordinates for a semi-detached binary system, including gas stream and accretion disk.

annulus of the disk maps to a ring on the Doppler map, the ring radii being projected Kepler velocities. Thus we get an "inside-out" image of the disk, with disk's outer rim mapping to the inner edge of the ring, and annuli closer to the disk center mapping to larger rings on the Doppler map. The ballistic trajectory of the gas stream maps to a curved arc on the Doppler map, starting from the L1 point, moving first toward $-v_{s}$ and then curving toward $-v_{y}$. A second arc, above the first, shows the Keplerian velocity ad different places along the stream.

Doppler maps of cataclysmic variables reveal a variety of structures, some expected and some unexpected (Kaitchuck et al. 1994). Doppler maps of quiescent dwarf novae show the expected ring of emission from the disk. Projections of this ring produce the double-peaked line profiles seen at all binary phases. An "S-wave" component often snakes back and forth between the two peaks. This arises from emission-line regions on the companion star and associated with the accretion stream and/or the impact region between the stream and the disk. It was a surprise that the $\mathrm{S}$-wave emission in $\mathrm{U}$ Gem arises not from the ballistic stream, nor from Keplerian gas at the position of the stream, but rather from gas with kinematics lying half-way between these two (Marsh et al. 1990). This suggests that two shocks form at the intersection of the hypersonic stream and disk flows, and the emission we see is in the post-shock region in which the two incoming flows are merged. Imaging the stream in $\mathrm{HI}, \mathrm{HeI}$, and HeII lines suggests that ionization structure of the stream-disk interaction region is on the verge of being resolved. At another epoch, the gas stream in $\mathrm{U}$ Gem is shut off, leaving just a clean ring of disk emission, plus an image of the companion star. The companion star image seen in H I and Ca II maps is elongated in the direction expected for the Roche lobe.

In AM Her stars the flow gets captured by magnetic field lines as it approaches the compact object. This gives rise to a ballistic stream flow, a transition region, and a magnetic curtain. X-rays emitted from the standoff shock at 

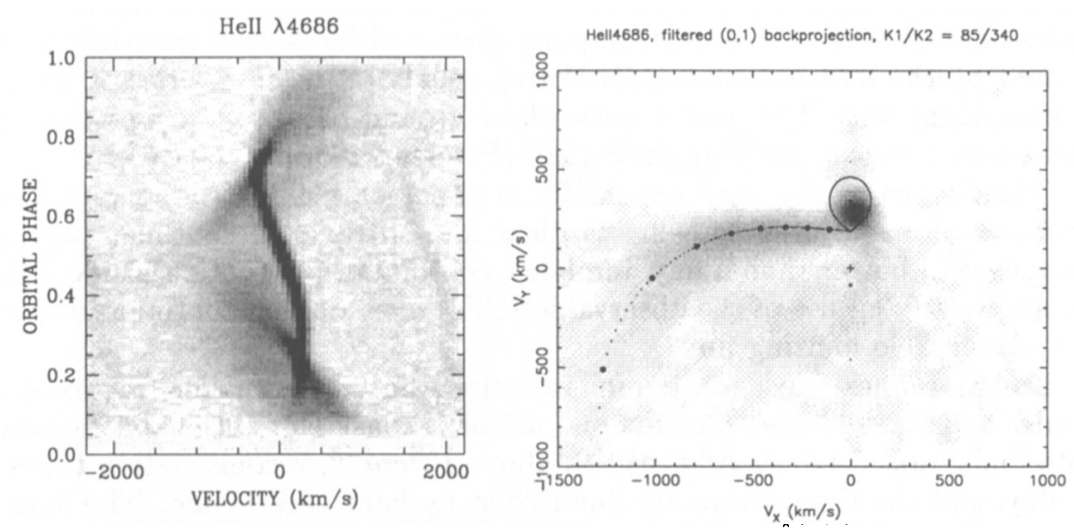

Figure 2. Trailed spectrogram data and Doppler map of He II 4686 emission in the magnetic cataclysmic variable $\mathrm{HU}$ Aqr showing X-ray irradiated face of the companion star and curved ballistic trajectory of the gas stream.

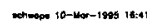

the foot of the flow illuminate the incoming flow, giving rise to the emission lines. Although the flow rises out of the orbital plane, the $v_{z}$ component of the motion is perpendicular to the line of sight and has no influence on the data in the case of eclipsing systems such as HU Aqr. Doppler maps of HU Aqr (Schwope et al. 1996, Fig. 2) reveal irradiation on the inward and trailing quadrant of the companion star, and in the curved ballistic stream emerging from the L1 point.

Doppler maps of SS Cyg and IP Peg in outburst show in addition to the disk and companion star, a nearly-stationary narrow emission component (Steeghs et al. 1996). While no part of the binary system has such a velocity, this feature can be interpreted as a "slingshot prominence", material between the L1 point and the white dwarf that is drawn by the white dwarf's gravity toward top of a magnetic loop anchored to the companion star.

A long-standing problem is the single-peaked emission lines seen in nova-like variables. These lines are deeply eclipsed, and exhibit rotational effects showing that the flow is circulating like a disk. However, the double peaks expected for disk lines are not seen. A solution is now offered by Murray and Chiang (1996), who show that single-peaked velocity profiles can arise from a Keplerian disk due to anisotropic emission in saturated lines formed in the outward acceleration zone at the base of a disk wind. The line escape probability is boosted in the direction of the acceleration, making the line emission stronger in the radial than in the azimuthal direction. This enhances line emission from the front and back of the disk surface, filling in the low-velocity core of the line profile. 


\section{Echo Mapping of AGN Emission-Line Regions}

Echo mapping exploits time delays and doppler shifts to probe the size, structure and kinematics of emission-line regions in the unresolved active nuclei of Seyfert galaxies (Blandford \& McKee 1982). The required time-resolved spectrophotometry with 1- to 4-day sampling sustained for several months has been acquired by the International AGN Watch collaboration in a series of monitoring campaigns with IUE and a network of ground-based telescopes that have focussed on 1 object per year since 1989 (Peterson 1993).

The Seyfert nuclei vary erratically in time, power density spectra having power-law slopes of -1 to -2 . Optical and ultraviolet continua vary synchronously to better than a day, implying continuum production inside 1 lightday, and justifying use of the observable IUEor even optical continua to trace of the unobservable ionizing flux.

Emission lines track continuum variations but with smaller fractional amplitudes and time-delayed maxima and minima, consistent with expectations for photo-ionization driving. At densities above $10^{8} \mathrm{~cm}^{-3}$, recombination times are $<1$ day, and the time delays are dominated by light travel time. The lines are delayed because the path from nucleus to photo-ionized gas to the observer is longer than the direct path from nucleus to observer.

By careful analysis of observed lightcurves and line profile variations, we recover a velocity-delay map $\Psi(v, \tau)$, the delay distribution of the emission-line response in each velocity bin. This resolves the emission-line region on iso-delay surfaces, which are paraboloids wrapped around the line of sight to the nucleus, and doppler shift surfaces, which depend on the unknown velocity field.

A maximum entropy fit recovers the velocity-delay map $\Psi(v, \tau)$, along with the driving continuum lightcurve $C(t)$, allowing for error bars and bridging gaps in the data as necessary. The line profile variations $L(v, t)$ are given by

$$
L(v, \tau)=\bar{L}(v)+\int_{\tau_{\min }}^{\tau_{\max }} \Psi(v, \tau)[C(t-\tau)-\bar{C}] d \tau
$$

were $\bar{L}(v)$ and $\bar{C}$ are time-steady line and continuum backgrounds. The $\chi^{2}$ statistic judges the fit. With $\bar{C}$ fixed at an arbitrary value, $\bar{L}(v), C(t)$, and $\Psi(\tau)$ are adjusted until $\chi^{2} / N=1$, where $N$ is the number of data points. The fit is not unique, but the maximum entropy criterion "steers" each point on $\bar{L}(v), C(t)$, and $\Psi(v, \tau)$ toward its neighbors, delivering the "smoothest" positive functions that fit the data. This method implemented in the computer code MEMECHO (Horne 1994).

Figure 3 shows a MEMECHO fit to simulated continuum and line lightcurves, recovering a delay map of the form $\Psi(\tau) \propto \tau e^{-\tau}$ in some detail. MEMECHO has been used to make delay maps of optical and ultraviolet emission lines in NGC 5548 (Horne, Welsh \& Peterson 1991, Krolik, et al. 1991, Wanders et al. 1995), Mrk 590 (Peterson, et al. 1993), NGC 3516 (Wanders \& Horne 1994), and NGC 4151 (Ulrich \& Horne 1996).

Figure 4 sketches the formation of the velocity-delay map for spherical freefall into a point-mass potential, and for a Keplerian disk (Welsh \& Horne 1991, Perez, Robinson \& de la Fuente 1992). The dramatically different appearance of the two images highlights the power of velocity-delay maps for kinematic diagnosis. 


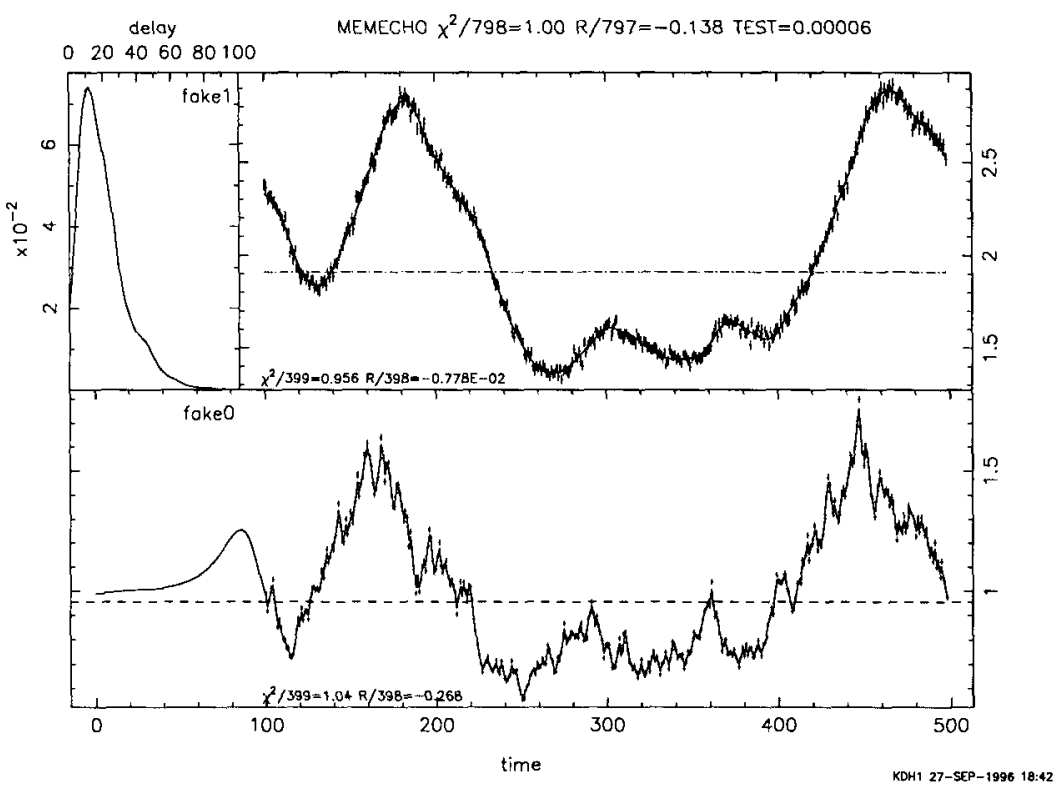

Figure 3. MEMECHO reconstruction of a 1-dimensional delay map from simulated lightcurves. The reconstructed map closely resembles the true map $\Psi(\tau) \propto \tau e^{-\tau}$.

Freefall

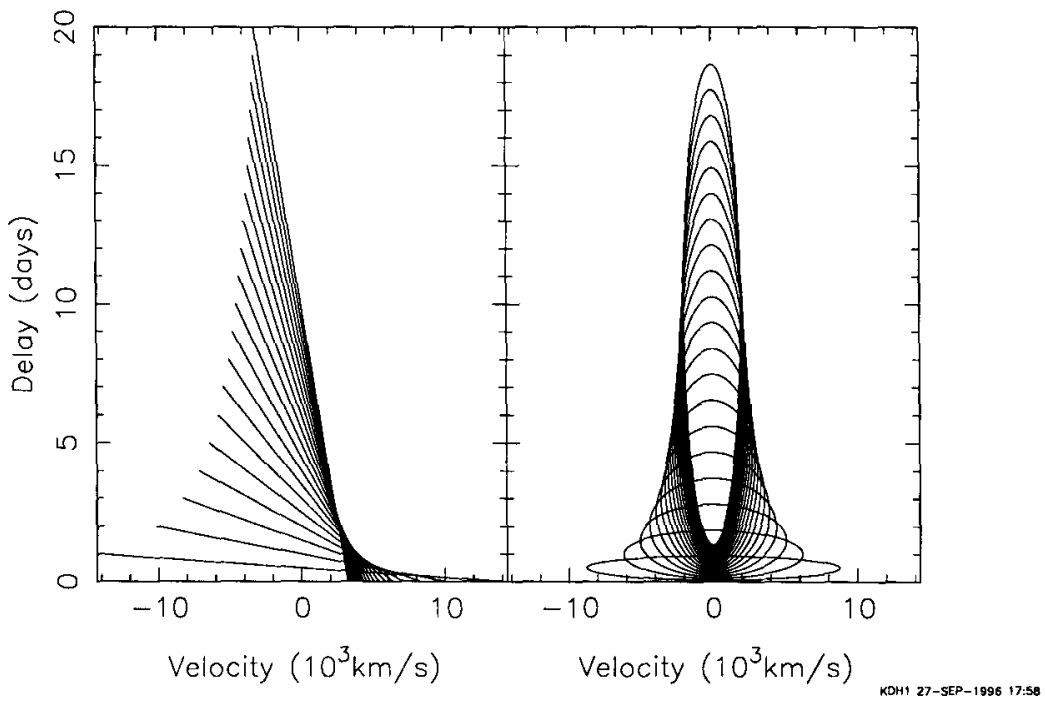

Figure 4. Velocity-delay maps for spherical freefall and Keplerian disk kinematics. Spherical shells map to diagonal lines, and disk annuli map to ellipses. The central mass is $10^{7} \mathrm{M}_{\odot}$ in both cases. The disk inclination angle is $60^{\circ}$. 
Any spherically-symmetric flow is a nested set of thin spherical shells. The time delay $\tau$ and doppler shift $v$ are

$$
\tau=\frac{2 R}{c}(1+\cos \theta), \quad v=v_{R} \cos \theta
$$

with $R$ the shell radius, $\theta$ the angle from the back edge, and $v_{R}$ the outflow velocity. The linear dependence of both $\tau$ and $v$ on $\cos \theta$ implies that each shell maps into a diagonal line in the velocity-delay plane, as shown in Figure 4.

The circulating disk flow is a set of concentric co-planar cylindrical annuli, for which the time delay and doppler shift are

$$
\tau=\frac{2 R}{c}(1+\sin i \sin \phi), \quad v=v_{\phi} \sin i \cos \phi,
$$

with $R$ and $i$ the radius and inclination of the annulus, $\phi$ the azimuth, and $v_{\phi}$ the azimuthal velocity. Each disk annulus maps to an ellipse on the velocity-delay map, as in Figure 4. Inner annuli map to "squashed" ellipses (large $\pm v$, small $\tau$ ), while outer annuli map to "stretched" ellipses (small $\pm v$, large $\tau$ ).

Even though the velocity-delay map projects a 6-dimensional phase space onto a 2-dimensional plane, it can still provide a good impression of flow when the velocity field is ordered and has some degree of symmetry. The envelope of the velocity-delay map can reveal the presence of virial motions $v \propto \sqrt{G M / c \tau}$, with $M$ the mass of the central object. A red/blue asymmetry or lack thereof can gauge the importance or absence of radial flow relative to azimuthal motions. A disordered velocity field with a range of velocities at each position smears the map in the $v$ direction. The far side of the map (large $\tau$ ) can be enhanced by anisotropic emission from optically thick clouds that radiate lines inward toward the nucleus (Ferland et al. 1992, O'Brien, Goad \& Gondhalekar 1994).

Figure 5 shows a velocity-delay map for CIV 1550 and HeII 1640 emission based on 44 IUE spectra of NGC 4151 covering 22 epochs during 1991 Nov 9 Dec 15 (Ulrich \& Horne 1996). Although spanning only 36 days, this campaign recorded favorable continuum variations, including a bumpy exponential decline followed by a rapid rise, which were sufficient to support echo mapping on delays from 0 to 20 days.

Disregarding the strong CIV absorption feature, which obliterates the delay structure at small velocities, the wide range of velocities at small delays and smaller range at larger delays suggests virial motions. The dashed curves in Figure 5 give escape velocity envelopes $v=\sqrt{2 G M / c \tau}$ for masses $0.5,1.0$, and $2.0 \times 10^{7} \mathrm{M}_{\odot}$, suggesting that a mass of order $10^{7} \mathrm{M}_{\odot}$ is concentrated within 1 light-day of the nucleus.

The approximate red-blue symmetry of the CIV map rules out pure inflow or outflow kinematics. The somewhat stronger CIV response on the red side at small delays and on the blue side at larger delays suggests a gas component with freefall kinematics. However, if the CIV emission arises from the irradiated faces of optically-thick clouds, those on the far side of the nucleus will be brighter, and the redward asymmetry can then be interpreted as an outflow combined with the inward CIV anisotropy.

Velocity-delay maps have been constructed for CIV emission in NGC 5548 (Wanders et al. 1995, Done \& Krolik 1996) and NGC 4151 (Ulrich \& Horne 1996). Both systems show the same trend of velocity dispersion decreasing with 


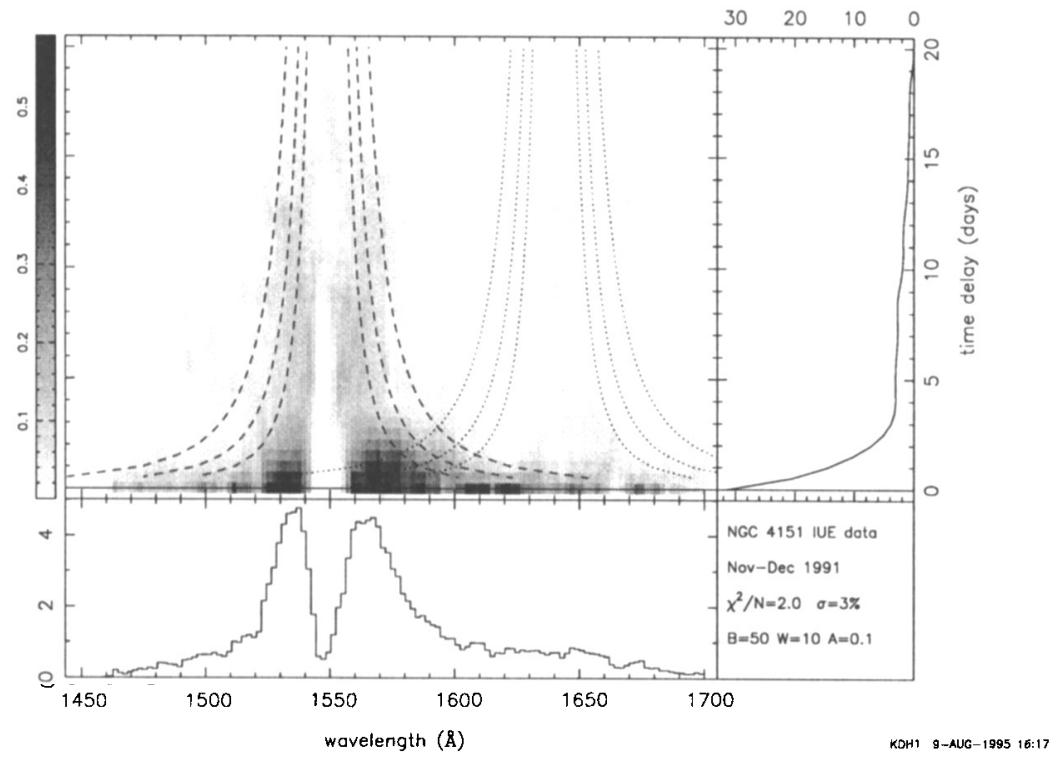

Figure 5. MEMECHO velocity-delay map of CIV 1550 emission (and superimposed HeII 1640 emission) in NGC 4151. Dashed curves give escape velocity for masses $0.5,1.0$, and $2.0 \times 10^{7} \mathrm{M}_{\odot}$.

delay, and red response stronger at small delays. As more maps are constructed we will see how well these features stand up as general characteristics of Seyfert broad-line regions.

\section{Physical Parameter Tomography}

At present most Astrotomography experiments construct intensity maps, either for continua at specific wavelengths, or for individual emission lines. This allows the data to speak as directly as possible, with a minimum of model-dependent assumptions. Intensity mapping is also a linear problem in the sense that the predicted data are linear functions of the image intensities. The interpretation of intensity maps can be complicated, however, because the maps suffer from artifacts that depend on the character and quality of the data. Tests with simulated data are usually required to decide which aspects of the maps are reliable.

With ever-faster computers, a new generation of Astrotomography codes is now being developed to make very general tests of specific physical models by simultaneous fitting of complete spectra, including the profiles of many lines and continua at many wavelengths, using maps of physical conditions in the flow. This should yield better maps with fewer artifacts because data at many wavelengths are brought to bear simultaneously on maps of a small number of physical parameters.

In cataclysmic variables, maps of the temperature, surface density, and velocity field should be obtainable by fitting to trailed-spectrogram datasets, 

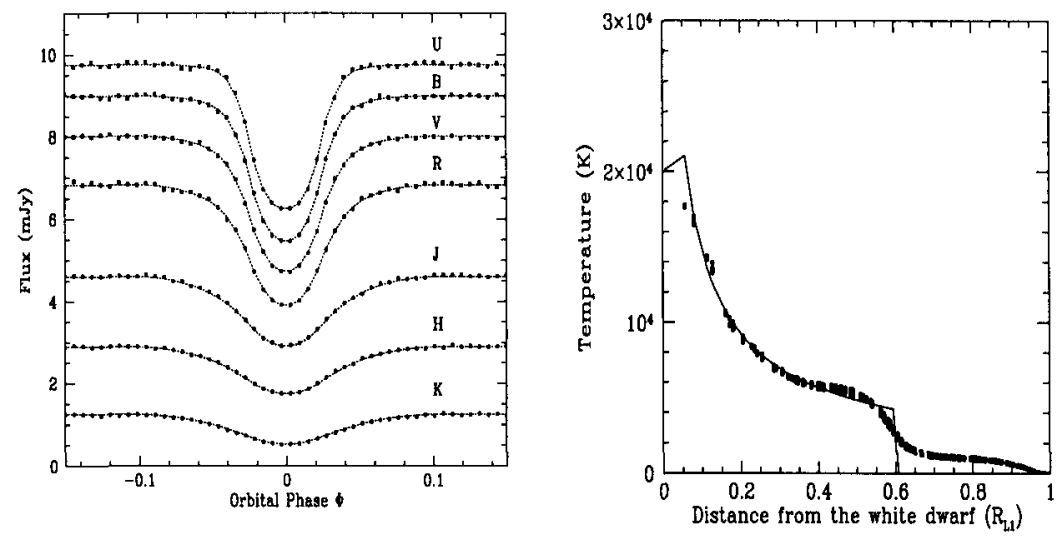

Figure 6. Simulated eclipse light curves in UBVRJHK bandpasses and comparison of the true and reconstructed temperature maps.

including eclipses and/or emission line profiles. A simple example (Vrielmann, these proceedings) is a temperature map fitted to simulated UBVRIJHK eclipse lightcurves (Fig. 6). Anisotropic turbulence (Horne 1995) and the acceleration zones of disk winds (Murray \& Chiang 1996) may be probed in this way.

For AGNs and other composite emission-line objects, photo-ionization models (e.g. CLOUDY) predict emissivities in dozens to hundreds of emission lines for gas clouds of specified density $n$, column density $N$, and incident ionizing flux $F$. Rather than fitting a model with 1 or 2 cloud types, we can use a full distribution function $f(n, N, F, v)$ on the 4-dimensional space spanning physical conditions and Doppler shift $v$, obtain predicted spectra by summing contributions over the full cloud distribution, then adjust $f(n, N, F, v)$ to fit observed spectra while steering toward a smooth distribution. The first applications of physical parameter tomography should be realized in the next few years.

\section{References}

Baptista, R., Horne, K., Hilditch, R. W., Mason, K. O., Drew, J. E. 1995, ApJ, 448,395

Baptista, R., Steiner, J. E., 1993, A\&A 277, 331

Blandford, R. D., Mc Kee, C. F. 1982, ApJ, 255, 419

Bruch, A., Beele, D., Baptista, R. 1996, A\&A, 306, 151

Done, C., Krolik, J. H. 1996, ApJ, 463, 144

Ferland, G. J., Peterson, B. M., Horne, K., Welsh, W. F., Nahar, S. N. 1992, ApJ, 387, 95 
Horne, K. 1993, "Eclipse Mapping of Accretion Disks: The First Decade", in Accretion Disks in Compact Stellar Systems, ed. J.C.Wheeler (World Scientific: Singapore), Advanced Series in Astronomy and Cosmology, 9, 117

Horne, K. 1994, "Echo Mapping Problems: Maximum Entropy Solutions", in Reverberation Mapping of the Broad Line Region in Active Galactic Nuclei, ed. P.M.Gondhalekar, K.Horne, B.M.Peterson (Astron.Soc.Pac: San Francisco), ASP Conf. Ser. 69, 23

Horne, K., 1995, A\&A, 297, 273

Horne, K., Cook, M. C. 1985, MNRAS, 214, 307

Horne, K., Marsh, T. R., 1986, MNRAS, 218, 761

Horne, K., Stiening, R. F. 1985, MNRAS, 216, 933

Horne, K., Welsh, W. F., Peterson, B. M. 1991, ApJL, 367, L5

Kaitchuck, R. H., Schlegel, R. M., Honeycutt, R. K., Horne, K., Marsh, T. R., White, J. C., Mansperger, C. S. 1994, ApJS, 93, 519

Krolik, J. H., Horne, K., Kallman, T. R., Malkan, M. A., Edelson, R. A., Kriss, G. A. 1991, ApJ, 371, 541

Lubow, S. H., Shu, F. H. 1975, ApJ, 198, 383

Marsh, T. R., Horne, K. 1988, MNRAS, 235, 269

Marsh, T. R., Horne, K., Schlegel, E. M., Honeycutt, R. K., Kaitchuck, R. H. 1990 ApJ, 364, 637

Murray, N., Chiang, J. 1996, Nature, 382, 789

O'Brien, P. T., Goad, M. R., Gondhalekar, P. M. 1994, MNRAS, 268, 845

Perez, E., Robinson, A., de la Fuente, L. 1992, MNRAS, 256, 103

Peterson, B. M., 1993, PASP, 105, 247

Peterson, B. M., Ali, B., Horne, K., Bertram, R., Lame, N. J., Poggee, R. W., Wagner, M., R. 1993, ApJ, 402, 469

Rutten, R. G. M., Dhillon, V. S., Horne, K., Kuulkers, E. 1994, A\&A, 283, 441

Rutten, R. G. M., van Paradijs, J., Tinbergen, J. 1992, A\&A, 260, 213

Schwope, A. D., Mantel, K.-H., Horne, K. 1996, A\&A, in press.

Steeghs, D., Horne, K., Marsh, T. R., Donati, J. F. 1996, MNRAS, 281, 626

Ulrich, M.-H., Horne, K. 1996, MNRAS, in press

Wanders, I., Horne, K. 1994, A\&A, 289, 76

Wanders, I., Goad, M. R., Korista, K. T., Peterson, B. M., Horne, K., Ferland, G., Koratkar, A. P., Poggee, R. W., Shields, J. C. 1995, ApJL, 453, L87

Welsh, W. F., Horne, K. 1991, ApJ, 379, 586

Wood, J. H., Horne, K., Berriman, G., Wade, R., 1989, ApJ, 341, 974

Wood, J. H., Horne, K., Berriman, G., Wade, R., O'Donoghue, D., Warner, B. 1986, MNRAS, 209, 629 


\section{Discussion}

P. Godon: Is the eclipse mapping (Doppler mapping) able to discover non axisymmetry, like eccentric discs, spiral patterns, ...?

K. Horne: Spiral patterns are hard to detect in eclipses (due to the need to steer toward an axi-symmetric map to find a unique solution) but should be easy to detect in Doppler mapping of the emission lines. Many such maps have been made, and none show spiral patterns.

B. Vaughan: Has a disk ever been observed in a $\mathrm{CV}$ with the opposite rotational sense of the binary?

K. Horne: No.

B. Vaughan: Would you be able to distinguish it?

K. Horne: Yes. 